\title{
Grosbeak: A Data Warehouse Supporting Resource-Aware Incremental Computing
}

\author{
Zuozhi Wang ${ }^{\dagger}$, Kai Zeng ${ }^{\ddagger}$, Botong Huang ${ }^{\ddagger}$, Wei Chen ${ }^{\ddagger}$, Xiaozong Cui ${ }^{\ddagger}$, Bo Wang ${ }^{\ddagger}$, \\ Ji Liu ${ }^{\ddagger}$, Liya Fan ${ }^{\ddagger}$, Dachuan $\mathrm{Qu}^{\ddagger}$, Zhenyu $\mathrm{Hou}^{\ddagger}$, Tao Guan ${ }^{\ddagger}$, \\ Chen $\mathrm{Li}^{\dagger}$, Jingren Zhou ${ }^{\ddagger}$ \\ $\dagger$ zuozhiw, chenli\}@ics.uci.edu, ${ }^{\ddagger}\{$ zengkai.zk, botong.huang, wickeychen.cw, xiaozong.cxz, yanyu.wb, niki.lj, \\ liya.fly, dachuan.qdc, zhenyuhou.hzy, tony.guan, jingren.zhou\}@alibaba-inc.com \\ ${ }_{\dagger}^{\dagger}$ UC Irvine, ${ }^{\ddagger}$ Alibaba Group
}

\begin{abstract}
As the primary approach to deriving decision-support insights, automated recurring routine analytic jobs account for a major part of cluster resource usages in modern enterprise data warehouses. These recurring routine jobs usually have stringent schedule and deadline determined by external business logic, and thus cause dreadful resource skew and severe resource over-provision in the cluster. In this paper, we present Grosbeak, a novel data warehouse that supports resource-aware incremental computing to process recurring routine jobs, smooths the resource skew, and optimizes the resource usage. Unlike batch processing in traditional data warehouses, Grosbeak leverages the fact that data is continuously ingested. It breaks an analysis job into small batches that incrementally process the progressively available data, and schedules these small-batch jobs intelligently when the cluster has free resources. In this demonstration, we showcase Grosbeak using real-world analysis pipelines. Users can interact with the data warehouse by registering recurring queries and observing the incremental scheduling behavior and smoothed resource usage pattern.
\end{abstract}

ACM Reference Format:

Zuozhi Wang, Kai Zeng, Botong Huang, Wei Chen, Xiaozong Cui, Bo Wang, Ji Liu, Liya Fan, Dachuan Qu, Zhenyu Hou, Tao Guan, Chen Li, Jingren Zhou. 2020. Grosbeak: A Data Warehouse Supporting Resource-Aware Incremental Computing. In Proceedings of the 2020 ACM SIGMOD International Conference on Management of

Permission to make digital or hard copies of all or part of this work for personal or classroom use is granted without fee provided that copies are not made or distributed for profit or commercial advantage and that copies bear this notice and the full citation on the first page. Copyrights for components of this work owned by others than ACM must be honored. Abstracting with credit is permitted. To copy otherwise, or republish, to post on servers or to redistribute to lists, requires prior specific permission and/or a fee. Request permissions from permissions@acm.org.

SIGMOD'20, fune 14-19, 2020, Portland, OR, USA

(c) 2020 Association for Computing Machinery.

ACM ISBN 978-1-4503-6735-6/20/06 ..\$15.00

https://doi.org/10.1145/3318464.3384708
Data (SIGMOD'20), June 14-19, 2020, Portland, OR, USA. ACM, New York, NY, USA, 4 pages. https://doi.org/10.1145/3318464.3384708

\section{INTRODUCTION}

Cloud-scale data warehouses are one of the keystone systems of a modern enterprise's big data infrastructure. They serve a mixed workload of recurring routine analytic jobs and ad-hoc analytic queries. As a primary approach to deriving decision-support insights, recurring routine analytic jobs account for a majority of cluster resource usages. For instance, from our observation of a data warehouse at Alibaba, up to $65 \%$ of daily workload is recurring analytic jobs. These jobs have a stringent schedule and deadline determined by various business logic. They also have similar schedules, thus causing dreadful "rush hour" schedule patterns in the cluster. Figure 1 shows a cluster resource usage pattern from a real traditional data warehouse environment in Alibaba. A majority of daily analysis queries starts after $2 \mathrm{AM}$ every day when the previous day's data has been fully collected. The peek usage lasts until 6AM, which is the deadline required by the business logic. During the day, there are only a few occasional spikes due to ad-hoc queries. This resource skew leads to a over-provision of resources to guarantee the peak resource usage, but leaves the cluster under utilized off traffic hours, causing excessive waste of resources.

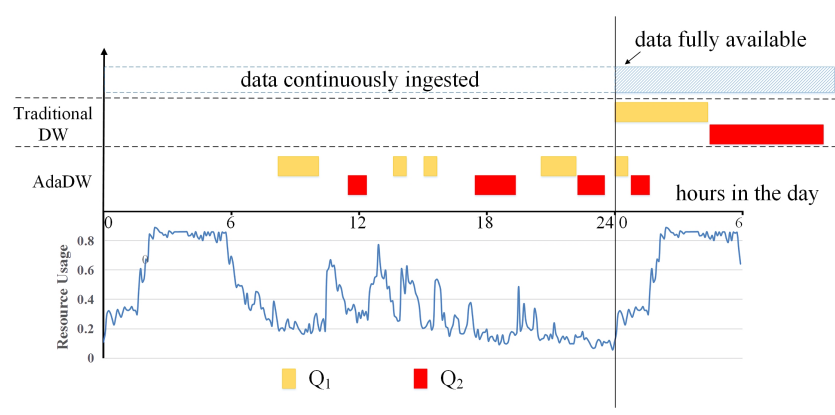

Figure 1: Timeline of running a pipeline of two queries in a traditional warehouse vs in Grosbeak 
To solve this problem, we have developed Grosbeak, a data warehouse that can automatically optimize the resource usage patterns of the underlying cluster. Specifically, Grosbeak focuses on optimizing the resource-usage pattern of recurring routine analytic jobs. Traditional data warehouses predominately handle automated routine analysis using batch query processing, and schedule them strictly at the time when all the input data is available. In contrast, Grosbeak leverages the fact that the analyzed data is continuously collected and ingested, and breaks an analysis job into small batches that incrementally process the progressively available data. These incremental jobs can be scheduled as part of the data becomes available, and can be adapted according to the real-time resource usage in the cluster.

To illustrate how recurring routine analytical jobs are executed in Grosbeak, let us consider an analytic pipeline where a query $Q_{1}$ computes on ingested data, whose output is in turn used by another query $Q_{2}$. Figure 1 illustrates how the pipeline is executed in a traditional data warehouse and in Grosbeak on a timeline. Traditionally, as the data is constantly ingested, the system cannot compute $Q_{1}$ and $Q_{2}$, even though the cluster has available capacities. Differently, Grosbeak can start computing $Q_{1}$ when part of $Q_{1}$ 's input data is available, and the cluster has available capacities (at 10am, 12pm, 18pm etc. of a day). Each execution can resume from the work left by the previous one, and the final execution at $12 \mathrm{am}$ of day 2 finishes much faster. Furthermore, the early outputs of $Q_{1}$ in turn enable $Q_{2}$ to execute early.

It is worth noting that dynamic scheduling in Grosbeak is very different from previous incremental computation paradigms, such as stream computing [2], incremental view maintenance [3] (IVM), etc. First, Grosbeak does not have a fixed triggering period. The scheduler can delay scheduling incremental computation if the cluster is overloaded by foreground ad-hoc workloads. In contrast, traditional IVM usually has to trigger incremental computation immediately to make sure its view is always up-to-date. Second, in between the incremental computation in Grosbeak, the recurring routine jobs do not occupy any cluster resources, and thus have much lower resource consumption. It is very different from paradigms such as stream computing, which has to keep the jobs online in order to process continuously arriving data. Third, the incremental execution in Grosbeak can be fully automated. Users do not need to manually convert their batch SQL queries into a streaming version. In addition, the data warehouse can search an optimal incremental plan from many incremental computing techniques, such as higher-order IVM etc. [1, 4] in a cost-based manner.

In this demonstration, we showcase Grosbeak using realworld analysis pipelines. Users can interact with the data warehouse by registering recurring queries, specifying hints for scheduling incremental processing, and observing the

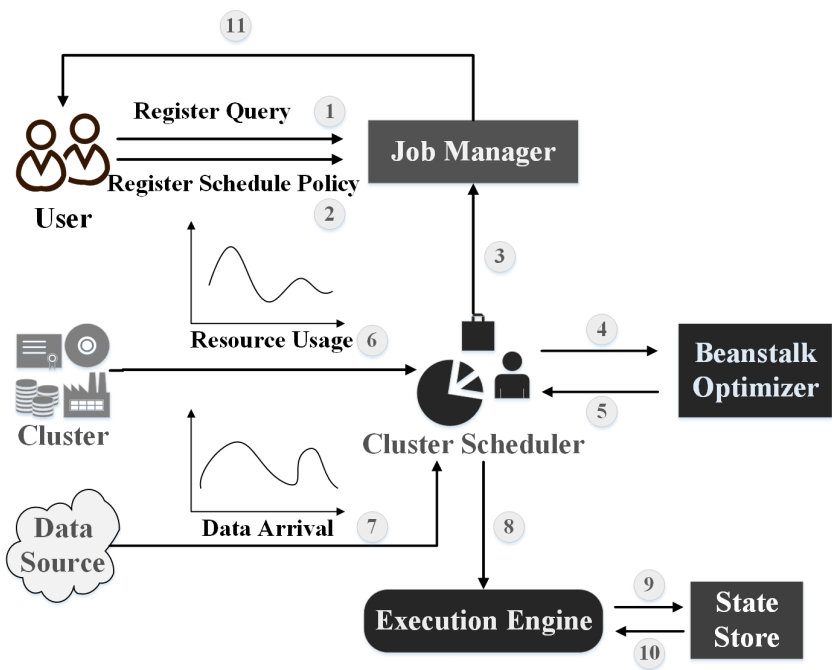

Figure 2: System architecture and job lifecycle

incremental scheduling behavior and the smoothed resource usage pattern in Grosbeak.

\section{GROSBEAK SYSTEM OVERVIEW}

Figure 2 shows the architecture of Grosbeak and the lifecycle of a job processed by the system. A user first registers a recurrent query on the fob Manager (step 1), including the query dependency in a pipeline and the deadline to produce the final result. The user can also specify a scheduling policy, such as triggering an execution when the cumulative input data passes a threshold. The user can also let Grosbeak automatically decide execution schedules. The fob Manager manages the whole query lifecycle and hosts the application UI. The query information is then passed to the Cluster Scheduler (step 3). The Cluster Scheduler generates a tentative schedule and invokes the Progressive-Plan Optimizer named Beanstalk (step 4), which uses cost-based optimization to generate a progressive plan (step 5). The plan consists of a sequence of physical execution plans to be executed at the scheduled times. The Cluster Scheduler starts monitoring the cluster resource usage (step 6) and data arrival for this query (step 7) in order to trigger the execution based on the cluster resource usage and user-provided scheduling policy. When an execution is triggered, the Cluster Scheduler submits the physical plan to the Execution Engine (step 8), which loads the previous intermediate states from the Grosbeak State Store (step 9), performs the incremental computation, and saves the new states (step 10). The State Store is a layered storage module that can intelligently choose a storage medium to keep the data, ranging from in-memory cache, SSD, to hard disks. This execution process (steps 8 - 10) repeats during the lifecycle of the job. The process ends when all the data has 
arrived, the last job finishes execution, and the final result is delivered back to the user (step 11).

When a job is initially submitted, the cluster scheduler proposes a tentative schedule $\{t 0, t 1, \ldots, t *\}$ and the optimizer generates corresponding progressive execution plans $\{P 0, P 1, \ldots, P *\}$. This scheduling decision is based on historical information and future predictions. During the lifecycle of the job, the cluster environment might change, causing the tentative schedule to be less ideal. Grosbeak supports dynamic re-planning to handle such situations. For example, if the cluster scheduler notices that the resource usage is high at an originally scheduled time, it will delay the execution and wait for the resource utilization to drop. The user could also manually set a new scheduling policy. When the Cluster Scheduler decides a new execution schedule, it repeats steps 4 and 5 to invoke the optimizer to perform re-optimization. The progressive scheduler produces a new plan by jointly considering the old partially executed plan, existing intermediate states, new schedule, and updated statistics. Specifically, users can specify the desired trigger time points, e.g., (12AM, 18PM, and 22PM), or a trigger condition, e.g., when the amount of accumulated data is beyond a threshold. Such hints can let users fine-tune the scheduling decisions based on external knowledge.

\section{QUERY COMPILATION}

Grosbeak uses a cost-based optimizer called "Beantalk" [5] that can jointly consider (1) various incremental view maintenance techniques, (2) the statistics of available data, (3) the current cluster resources, and (4) the intermediate states from the previous runs and the schedules of the incremental jobs in the future. In this section, we present Grosbeak's compilation technique through an example, illustrate the plan space of various ways of incremental processing, and demonstrate the cost trade-off. We propose a general framework based on a model called "time-varying relation (TVR) model", in which we treat changing data as a relation varying with time. By explicitly modeling snapshots and delta views of a TVR, we can unify various incremental computation models. We elaborate the complexity of progressive query planning using an example analytic pipeline in Fig. 3(a), which computes the gross revenue of all orders by consolidating the sales orders with the returned ones. Suppose we are given a schedule $S$ with two times $t_{1}$ and $t_{2}$, where $t_{2}$ is the specified execution time of both queries in the workflow, and $t_{1}$ is an early execution time. The data records visible at $t_{1}$ and $t_{2}$ in sales and returns are those in Fig. 3(b).

Classic incremental view maintenance. This approach treats sales_status and summary as views, and uses incremental computation to keep the views always up to date with respect to the data seen so far. At time $t_{1}$, it computes the left outer join using the snapshot at $t_{1}$ of sales and returns to generate a view sales_status, then applies aggregation on the join result to generate a view summary. At time $t_{2}$, instead of recomputing from scratch, it uses the delta input to incrementally compute the result. It first computes the delta of the left outer join by using the snapshot at $t_{1}$ and $\operatorname{delta}\left(t_{1}-t_{2}\right)$ of sales and returns, then performs aggregation on join's delta outputs. The delta outputs are merged with previous snapshots to update the two views. The delta input to sales at $t_{2}$ includes tuples $\left\{o_{5}, o_{6}, o_{7}\right\}$. Fig. 3(c) depicts sales_status's delta outputs at $t_{1}$ and $t_{2}$, respectively, where $\#=+/-1$ denotes insertion or deletion, respectively. Note that a returns record (e.g., $r_{2}$ at $t_{2}$ ) can arrive much later than its corresponding order (e.g., the shaded $o_{2}$ at $t_{1}$ ). Therefore, an order record may be output early as it cannot join with a returns record at $t_{1}$, but retracted later at $t_{2}$ when the returns record arrives, such as tuple $o_{2}$ of sales_status at $t_{2}$ in Fig. 3(c). Non-retractable incremental computation. The approach avoids retractions in incremental computation. Specifically, in the outer join of sales_status, tuples in sales that do not join with tuples from returns at $t_{1}\left(o_{2}, o_{3}\right.$, and $\left.o_{4}\right)$ may join in the future, and thus will be held back. At time $t_{1}$, it only computes the inner join on snapshot $\left(t_{1}\right)$ of sales and returns and applies the aggregation. The computation of left anti join is skipped because it produces outputs that could possibly be retracted. At time $t_{2}$, it first computes the delta of sales $\bowtie$ returns and incrementally computes the aggregation. Because $t_{2}$ is the last execution time and all data has arrived, $\gamma\left(\right.$ sales $\bowtie^{l a}$ returns) can be computed, then merged with the inner join aggregation result to produce the final output.

The optimal progressive plan is data dependent, and should be determined in a cost-based way. In this example, the classic IVM approach computes 9 tuples (5 tuples in the outer join and 4 tuples in the aggregate) at $t_{1}$, and 10 tuples at $t_{2}$. Suppose the cost per unit at $t_{1}$ is 0.2 (due to fewer queries), and the cost per unit at $t_{2}$ is 1 . Then its total cost is $9 \times 0.2+10 \times 1=11.8$. The non-retractable incremental computing approach computes 6 tuples at $t_{1}$, and 11 tuples at $t_{2}$, with a total cost of $6 \times 0.2+11 \times 1=12.2$. On the contrary, if the retraction is often, say, with one more order tuple $o_{4}$ at $t_{2}$, then the stream computing approach will become more efficient, as it costs 12.2 versus the cost 13.8 of the first approach. The reason is that retraction wastes earlier computation and causes more re-computation. Notice that the performance difference of the two approaches can be arbitrarily large.

\section{DEMONSTRATION SCENARIO}

This demonstration will allow the audience to interact with Grosbeak using a variety of data sets and queries, such as those from the TPC-DS benchmark. We will also provide data 


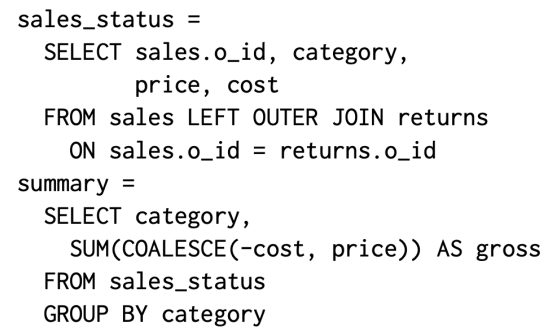

(a)

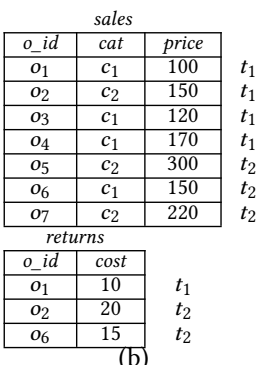

(b)
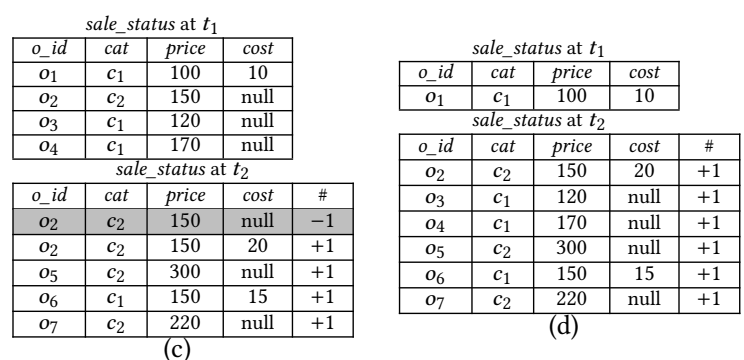

(d)

Figure 3: (a) A simple analytic pipeline; (b) data-arrival patterns of sales and returns; (c) results of sales_status produced by IVM at $t_{1}$ and $t_{2}$; and (d) results of sales_status produced by non-retractable computing at $t_{1}$ and $t_{2}$.

sets and query workloads that closely resemble the business analytics jobs in Alibaba.

Generating reports for an online retailing business. We demonstrate a real use case of Grosbeak in an online retailing business, which involves processing of orders, payment, and logistics data to generate daily reports. Such reports are crucial to supporting business intelligence and decision making. Data is continuously ingested into the warehouse by synchronizing with the online OLTP database change log every 15 minutes. One example application is to generate shipping status of all orders placed in a day. The query uses a left outer join of orders with logistics on the order_id attribute. Left outer join is used because only orders that are shipped out on the same day as they are placed can successfully join. An orders record is inserted immediately after an order is placed, but a logistics record is only created after a tracking number is available. In this case, Grosbeak can intelligently choose the non-retractable incremental computation approach described in Section 3 to avoid a lot of retractions. Another interesting characteristic of this workload is that most of the data arrival happens during the day, which does not coincide with the peak hours (2AM - 6AM) of the cluster. Grosbeak is able to choose an execution schedule without accumulating too much data.

Monitoring status on application dashboard. Grosbeak provides an application UI that helps users understand the progressive plans and monitor the status of the job lifecycle. Figure 4 shows a query dependency graph consisting of four queries. The user can see each query with two or three scheduled executions and the start and finish times of each execution. The user can further click each job to inspect the job detail, including the physical plan at each time point, the intermediate states saved by each job, and the early outputs to downstream queries in the pipeline. When the scheduler triggers a job, the UI updates the run-time execution detail of each job. The user can inspect the current execution status of the query, including the amount of early data processed and the amount of early outputs to downstream queries.

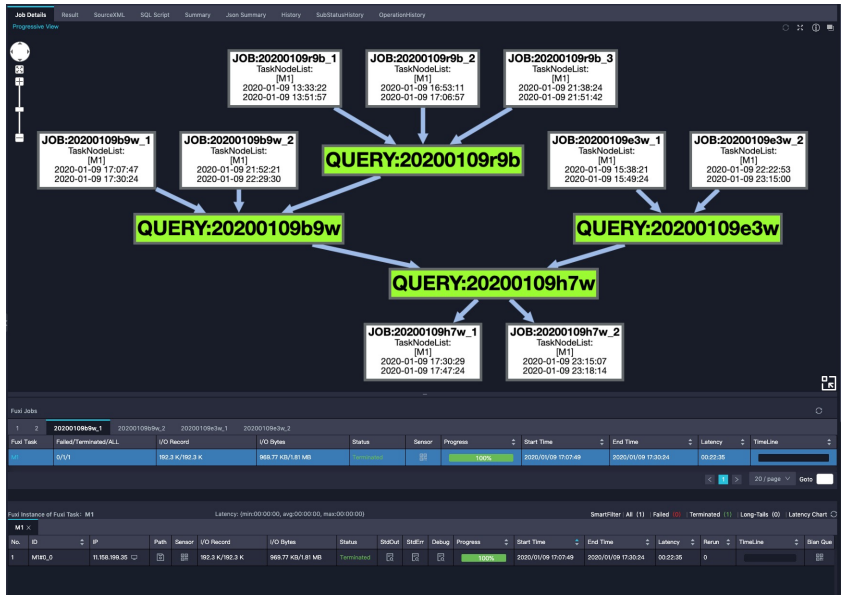

Figure 4: Application UI in Grosbeak

\section{REFERENCES}

[1] Yanif Ahmad, Oliver Kennedy, Christoph Koch, and Milos Nikolic. 2012. Dbtoaster: Higher-order delta processing for dynamic, frequently fresh views. PVLDB 5, 10 (2012), 968-979.

[2] Arvind Arasu, Shivnath Babu, and Jennifer Widom. 2006. The CQL continuous query language: semantic foundations and query execution. The VLDB fournal 15, 2 (2006), 121-142.

[3] Surajit Chaudhuri, Ravi Krishnamurthy, Spyros Potamianos, and Kyuseok Shim. 1995. Optimizing Queries with Materialized Views. In Proceedings of the Eleventh International Conference on Data Engineering (ICDE '95). IEEE Computer Society, Washington, DC, USA, 190-200. http://dl.acm.org/citation.cfm?id=645480.655434

[4] Per-Åke Larson and Jingren Zhou. 2007. Efficient Maintenance of Materialized Outer-Join Views. In Proceedings of the 23rd International Conference on Data Engineering, ICDE 2007, The Marmara Hotel, Istanbul, Turkey, April 15-20, 2007. 56-65. https://doi.org/10.1109/ICDE.2007. 367851

[5] Planning Progressive Execution: Resource Smoothing in Cloud-Scale Data Warehouses [n. d.]. Technical Report, https://kai-zeng.github.io/ papers/beanstalk-tech-report.pdf. 